\title{
Static and Dynamic Disorder in Triple-Cation
}

\section{Hybrid Perovskites}

\author{
M. Baranowski, ${ }^{\dagger} \ddagger$ J. M. Urban, ${ }^{\dagger}$ N. Zhang, ${ }^{\dagger}$ A. Surrente, ${ }^{\dagger}$ D. K. Maude, ${ }^{\dagger}$ Zahra $^{\prime}$ \\ Andaji-Garmaroudi, S. D. Stranks, "and P. Plochocka*,† \\ $\dagger$ Laboratoire National des Champs Magnétiques Intenses, UPR 3228, \\ CNRS-UGA-UPS-INSA, Grenoble and Toulouse, France \\ $\ddagger$ Department of Experimental Physics, Faculty of Fundamental Problems of Technology, \\ Wroclaw University of Science and Technology, Wroclaw, Poland \\ \Cavendish Laboratory, J.J. Thomson Avenue, Cambridge CB3 OHE, United Kingdom \\ E-mail: paulina.plochocka@lncmi.cnrs.fr
}




\begin{abstract}
A detailed understanding of the carrier dynamics and emission characteristics of organic-inorganic lead halide perovskites is critical for their optoelectronic and energy harvesting applications. In this work, we reveal the impact of the crystal lattice disorder on the photo-generated electron-hole pairs through low-temperature photoluminescence measurements. We provide strong evidence that the intrinsic disorder forms a sub-bandgap tail density of states, which determines the emission properties at low temperature. The PL spectra indicate that the disorder evolves with increasing temperature, changing its character from static to dynamic. This change is accompanied by a rapid drop of the PL efficiency, originating from the increased mobility of excitons/polarons, which enables them to reach deep non-radiative recombination centers more easily.
\end{abstract}

\title{
INTRODUCTION
}

Organic-inorganic perovskites have emerged as an extremely promising class of materials for energy harvesting applications. Perovskite based solar cells recently exceeded 20\% power conversion efficiency, $\stackrel{112}{ }$ with an unprecedented fivefold increase in less than 10 years. $\stackrel{3}{3}$ This has led to a renewed interest in understanding their basic physical properties, which are responsible for the outstanding performance of these optoelectronic devices. The combination of a large optical absorption coefficient, long carrier lifetime and diffusion length in these materials, ${ }^{4} 6$ along with the simple and cheap fabrication with a minimal energetic footprint, makes organic-inorganic perovskites ideal candidates for both photovoltaic and light emitting applications. $\frac{718}{}$

In organic-inorganic perovskites, the methylammonium $\left(\mathrm{CH}_{3} \mathrm{NH}_{3}{ }^{+}, \mathrm{MA}\right)$ or formamidinium $\mathrm{CH}\left(\mathrm{NH}_{2}\right)_{2}{ }^{+}, \mathrm{FA}$ ) cation is surrounded by $\mathrm{PbX}_{6}$ octahedra. The band-edge states are composed essentially from the orbitals of the $\mathrm{Pb}$ and halide atoms. ${ }^{9} 11$ The conduction band is composed of $\mathrm{Pb}$ p-type orbitals while the valance band is composed of $\mathrm{Pb}$ s-type orbital 
with admixture of p-type halide orbitals. However organic cations also play an important role in the electronic and optical properties of organic-inorganic perovskites. ${ }^{121}$ The motion of the organic cation and its local arrangement affect both the lattice parameters and the internal geometry of the crystal, lowering its symmetry $\stackrel{20}{20}$ at tetragonal and orthorhombic phase and locally breaking the symmetry on ps-time scale. ${ }^{22}$ The wide range of possible bond angles between the organic cations and the halide cage gives rise to a plethora of distortions of

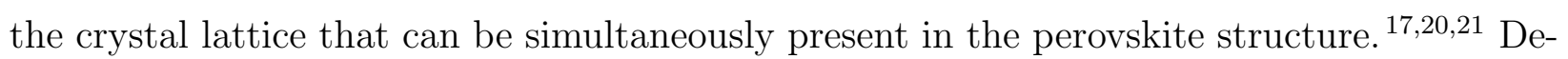
tailed density functional theory calculations combined with molecular dynamics simulations reveal that these distortions affect the position of the bands and the local density of states. The resulting fluctuations in the potential landscape have been proposed to explain the modest carriers mobilities $\frac{19}{19}$ and can even change the nature of the fundamental band edge

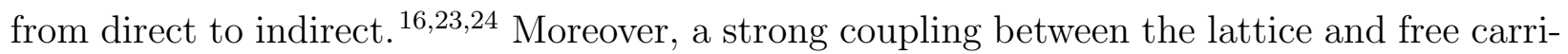
ers/excitons can lead to local screening of carriers and the formation of polarons. $\frac{13 \mid 22[25 \sqrt{30}}{1 n}$ the high temperature phase ( $T>160 \mathrm{~K}$ for MA and $T>120 \mathrm{~K}$ for $\mathrm{FA})$, the organic cations are free to rotate, and the induced disorder has a dynamic nature.17/19/20|24|31|32 At lower temperatures, the motion of organic cations becomes progressively more restricted.12[14]32 34 Nevertheless, the temporary disordered configuration of MA or FA can be transferred to the low temperature orthorhombic phase, which leads to the formation of distorted orthorhombic domains ${ }^{12}$ or even a "frozen" tetragonal phase. ${ }^{\sqrt[35 \mid 36]{36}}$ Thus, the presence of organic cations leads to an intrinsic disorder in the organic-inorganic lead halide perovskites, in addition to extrinsic disorder due to alloying or grain boundaries.

In this work, we use low-temperature photoluminescence (PL) and transmission spectroscopy to elucidate the basic photo-physical properties of the state-of-the-art triple cation

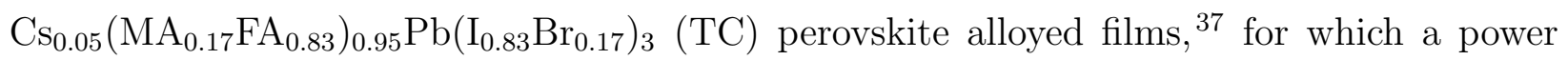

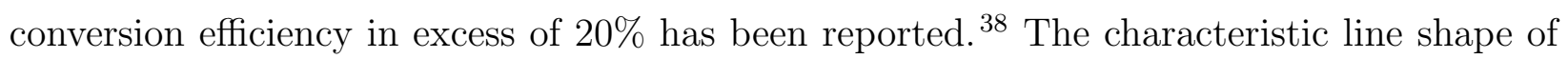
the PL indicates that the low temperature emission is dominated by carriers/excitons localized by shallow trapping states forming an exponentially vanishing tail density of states 
below the band edge. We find that the PL points to an evolution of the trapping state population with increasing temperature. Our results demonstrate a change in the character of the disorder from static to dynamic with increasing temperature. This is accompanied by a rapid drop of the PL intensity. In light of this, we discuss the fundamental impact of the below band gap states on the recombination process and their potential nature.

\section{METHODS}

Cover slips were cleaned by sonication in acetone and isopropyl alcohol for 30 min, then the substrates were further cleaned with an oxygen plasma treatment for $10 \mathrm{~min}$. The $\mathrm{Cs}_{0.05}\left(\mathrm{MA}_{0.17} \mathrm{FA}_{0.83}\right)_{0.95} \mathrm{~Pb}\left(\mathrm{I}_{0.83} \mathrm{Br}_{0.17}\right)_{3}$ perovskite precursor solution was prepared by dissolving $\mathrm{PbI}_{2}(1.1 \mathrm{M}), \mathrm{PbBr}_{2}(0.22 \mathrm{M})$, formamidinium iodide (1M), and methylammonium bromide $(0.20 \mathrm{M})$ in a mixture of anhydrous DMF:DMSO (4:1 volume ratio, v:v) followed by addition of 5 vol\% from CsI stock solution (1.5M in DMSO). The perovskite solution was spin-coated on the glass substrates in a two-step program at 1000 and $4000 \mathrm{rpm}$ for 10 and $30 \mathrm{~s}$ respectively, and $110 \mu \mathrm{l}$ of chlorobenzene was poured on the spinning substrate $30 \mathrm{~s}$ after the starting of the program. The substrates were then annealed at $100{ }^{\circ} \mathrm{C}$ for 1 hour. Synthesis and deposition of perovskite solutions were performed inside a nitrogen glove box under controlled moisture (5pm) and oxygen conditions (0.5ppm).

For optical spectroscopy, the samples were mounted in an exchange gas helium cryostat. Optical access to the samples was provided through a quartz window. The PL measurements were performed in backscattering configuration. The sample was excited by a pulsed laser tuned to $540 \mathrm{~nm}$, with a pulse duration of $300 \mathrm{fs}$. The excitation beam was focused on the sample by a lens having a focal length of $20 \mathrm{~cm}$. The same lens was used to collect the PL signal, which was analyzed using a $50 \mathrm{~cm}$ focal length spectrometer equipped with a 600 grooves/mm grating and detected using liquid nitrogen cooled Si CCD camera. The transmission measurements were performed in the same setup, using the white light generated by 


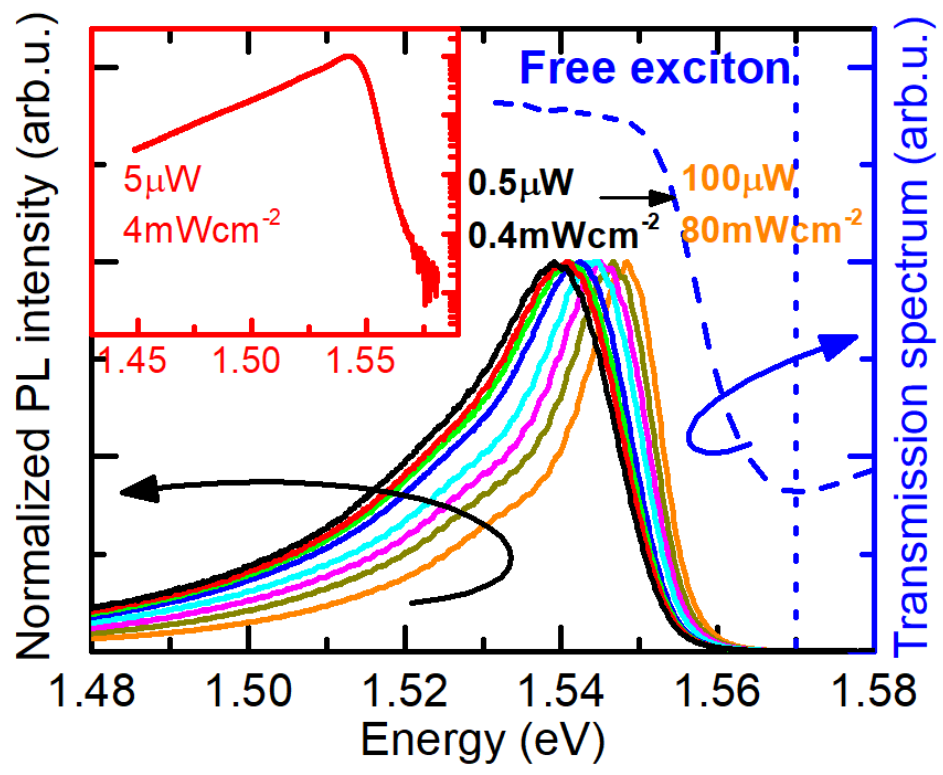

Figure 1: Full lines: PL spectra of $\mathrm{Cs}_{0.05} \mathrm{MA}_{0.16} \mathrm{FA}_{0.7} 9 \mathrm{~Pb}\left(\mathrm{I}_{0.83} \mathrm{Br}_{0.17}\right)_{3}$ thin films showing blue shift with increasing excitation power. Dashed blue line: transmission spectrum measured at the same position of the sample as PL spectrum. The PL spectra are measured with $0.5 \mu \mathrm{W}, 1 \mu \mathrm{W}, 2 \mu \mathrm{W}, 5 \mu \mathrm{W}, 10 \mu \mathrm{W}, 20 \mu \mathrm{W}, 50 \mu \mathrm{W}, 100 \mu \mathrm{W}$ excitation powers. The excitation wavelength is $540 \mathrm{~nm}$. Inset: PL spectrum plotted in logarithmic scale, revealing the low energy exponential tail. Measurements were performed at $1.5 \mathrm{~K}$.

a halogen lamp as broadband light source. The signal for time resolved PL measurements was dispersed by a $30 \mathrm{~cm}$ focal length monochromator with 300 grooves $/ \mathrm{mm}$ grating, and detected using a streak camera.

\section{RESULTS \& DISCUSSION}

Figure 1 shows representative PL spectra measured at $T=1.5 \mathrm{~K}$ at different excitation powers, together with a transmission spectrum measured at the same position of the sample. The PL has a characteristic shape with a relatively sharp cut off on high energy side, and a long exponential tail on the low energy side, as shown more clearly in the semilogarithmic plot of the inset of Fig. 1. The PL peak is red shifted from the dip in the transmission spectrum, which corresponds to the free exciton (FX) absorption. ${ }^{39} 42$ The magnitude of this red shift depends on the excitation power, and gradually decreases with increasing power, while the PL 
line shape is unaffected. This behavior is characteristic of PL associated with an exponential tail of density of states, as reported for inorganic semiconductors. ${ }^{43}$ [46 In semiconductors, the carrier/exciton relaxation rates are generally much higher than the recombination rates, so the photo-created excitons thermalize (that is, are trapped to form localized excitons) before radiative recombination. In contrast, the absorption probes the joint density of states, and therefore its resonance corresponds to the free exciton (FX) transition. The different time scale of relaxation and recombination explains the shift observed between the PL peak and the FX absorption energy as well as it power dependence. The continuous shift of the PL maximum indicates that the localized states form a continuous distribution of energy levels - a band tail ${ }^{47}$ - rather than a single localized level. The exponential decrease of the PL spectrum at low energies shown in the inset of Fig. 1 is a direct evidence for the exponential distribution with energy of the trapping states.

The process of carrier relaxation through a tail density of states can be directly observed in time resolved PL measurements. Because the relaxation processes are much faster than recombination, this process is most prominent in the first few tens of ps of the PL temporal evolution. Figure 2(a) shows a streak camera image of the temporal evolution of the PL, monitored up to $200 \mathrm{ps}$ after the excitation pulse, and measured at $T=1.5 \mathrm{~K}$. Characteristic features of PL dynamics in disordered systems ${ }^{\frac{48149}{4}}$ can be seen: (i) The PL maximum shifts to lower energy with time (see Fig. 2(b)), and (ii) there is a strong energy dispersion of the PL decay as function of energy (see Fig. 2(c)). The streak image very nicely illustrates the process of carrier relaxation through the population of the localized states. Immediately after excitation, the PL peak is situated only a few meV below the energy of the free exciton, determined from the transmission spectrum (see Fig. 2(a,b)), and after red shifts continuously with time. The probability of free excitons being captured by trapping states with a given energy is proportional to the density of states. Therefore, just after excitation the PL spectrum reflects the shape of the tail of density states, and the PL peak is close to the free exciton energy (Fig. 2 (b)). The later redshift of the PL maximum is induced by 
the thermalization of excitons to unoccupied, deeper states, i.e., driven by the tendency of the exciton population to reach thermal equilibrium with the lattice. Excitons trapped on shallow states act as a reservoir, which progressively populates the deeper states leading to a marked asymmetry of the PL decay time. It is important to note that the PL originating from donor-acceptor recombination can posses very similar characteristic features, $\frac{50}{2 . e . ~ s t r o n g}$ Stokes shift dependence as a function of the excitation power and PL decay time asymmetry. However, we think that the strong dependence of the decay time at a given energy of detection on the excitation power (see Supporting Information) clearly points to the transfer process between localized states. Therefore, the application of the tail density of states picture is more accurate than the donor-acceptor model.

Importantly, our time resolved measurements performed at $T=1.5 \mathrm{~K}$ do not reveal any signature of hot photoluminescence previously reported at room temperatures. $26151+53$ The motion of organic cations at room temperature results in the "dressing" of the electrons and holes by the local organic sublattice polarization. This protects carriers from efficient scattering by longitudinal optical (LO) phonons, $\stackrel{25 \mid 26}{\sqrt{26}}$ elongating the carrier relaxation to the band minimum and hot PL can be observed for few hundreds of ps up to a nanosecond after excitation. $\frac{26151+53}{53}$ Our results demonstrate that at low temperatures, even very shortly after excitation, all the emitted photons have an energy below the free exciton transition. This indicates that the organic cation motion is frozen, which reduces the dynamic carrier screening. ${ }^{26}$ Therefore, LO phonon scattering can efficiently cool the photo-induced carriers down, in a similar fashion to III-V semiconductors. $\underline{54}$

Our PL data can be qualitatively explained using a hopping model previously employed for inorganic disordered semiconductors. 4455 The schematic of the model is presented in Fig. 3(a) and (b). The model assumes that excitons behave like non-interacting single particles trapped by local potential minima with the possibility of excitons hopping between different states, which have an exponentially decreasing density of states with decreasing energy. Non-radiative recombination is neglected, as we focus on the PL line shape rather 

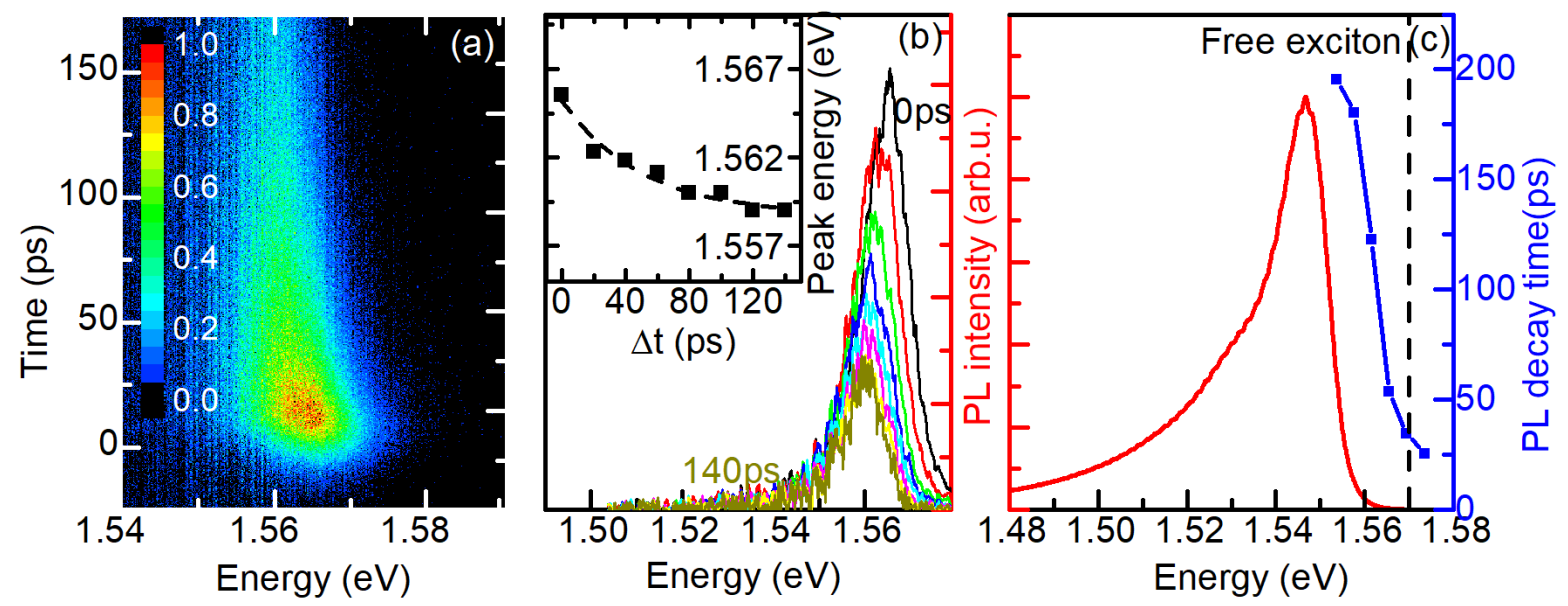

Figure 2: (a) Streak camera image of initial 200 ps temporal dependence of the PL measured at $T=1.5 \mathrm{~K}$ with $50 \mu \mathrm{W}$ excitation power and $540 \mathrm{~nm}$ excitation wavelength. (b) PL spectra at different times after excitation pulse (20 ps step). Inset: PL peak position as a function of time. (c) Time integrated PL spectrum measured with $50 \mu \mathrm{W}$ excitation power (red curve) together with decay time constant energy dispersion (blue curve). The decay time is determined as the temporal distance between PL intensity maximum and the value where the PL intensity decreases by a factor of e.

than the intensity and the time scales of nonradiative recombination $(\sim 1-100 \mathrm{~ns})$ are much slower than hopping and trapping processes ( 1-100 ps). A trapped exciton can either recombine radiatively, hop to another localized state or eventually be activated to a free exciton state (mobility edge which provides the zero reference for energy in the model). The dynamics of excitons (PL) are determined by the rates of the competing processes. The hopping rate is described by the Miller-Abrahams formula: $\underline{4462}$

$$
v_{i j}=v_{0} \exp \left(-\frac{2 r_{i j}}{\alpha}\right) \exp \left(\frac{E_{i}-E_{j}}{k_{\mathrm{B}} T}\right),
$$

if $E_{j}>E_{i}$ and

$$
v_{i j}=v_{0} \exp \left(-\frac{2 r_{i j}}{\alpha}\right),
$$

if $E_{j} \leq E_{i}$. Here, $v_{i j}$ is the hopping rate between states $i$ and $j$ with energy $E_{i}$ and $E_{j}, v_{0}$ is an attempt rate, $\alpha$ is the decay length of the exciton wave function and $T$ indicates the 
temperature. The rate of exciton activation to the mobility edge (free exciton) is

$$
v_{\text {act }}=v_{0} \exp \left(-\frac{E_{i}}{k_{\mathrm{B}} T}\right),
$$

and the radiative recombination rate is simply the inverse of exciton radiative life time $v_{\text {rec }}=\tau_{\mathrm{r}}^{-1}$. The simulation of exciton dynamics is performed on a randomly generated set of trapping sites (within a cubic volume of size $L^{3}$ with periodic boundary conditions) with uniform position distribution and exponential energy distribution with a density of states

$$
D(E)=\frac{N}{L^{3} E_{0}} \exp \left(-\frac{E}{E_{0}}\right)
$$

where $E_{0}$ is the average localizing energy and $N$ is the number of traps in a cube of size $L \times L \times L$. The details of the approach used for the simulations can be found in the Supporting Information and in previous works. $63[64$ The carrier dynamics predicted by the model and the PL spectrum are determined by the dimensionless parameters $\tau_{\mathrm{r}} v_{0}$, and $\frac{N_{0} \alpha^{3}}{L^{3}}$, which can be treated as an effective density of states, and $E_{0}$. The average energy of localization can be estimated from the observed PL spectral broadening, which depends weakly on other parameters. $\frac{65666}{6 t}$ At very low temperature $\left(\mathrm{k}_{\mathrm{B}} T \ll E_{0}\right)$, the full width half maximum (FWHM) of PL spectrum is FWHM $\sim 2.5 E_{0}$. In our case, this leads to $E_{0} \approx 14 \mathrm{meV}$. We note that this value matches quite closely the experimentally measured values for the Urbach energy in related systems. $\underline{67 / 68}$ The other parameters used in the simulation are $\tau_{\mathrm{r}} v_{0}=2 \times 10^{4}$ and $\frac{N_{0} \alpha^{3}}{L^{3}}=0.088$ (see Supporting Information for more details). The PL intensity is assumed to be proportional to the number of occupied states at that energy at any given time.

The simulated PL spectra are presented in Fig.3(c). For the chosen parameters, the shape of the simulated PL (solid lines) reproduces very nicely the measured PL spectra (symbols). The power-dependent shift of the PL peak as well as their temporal characteristics, such as decay time dispersion (green circle) and PL peak shift with time (inset), are 
(a)

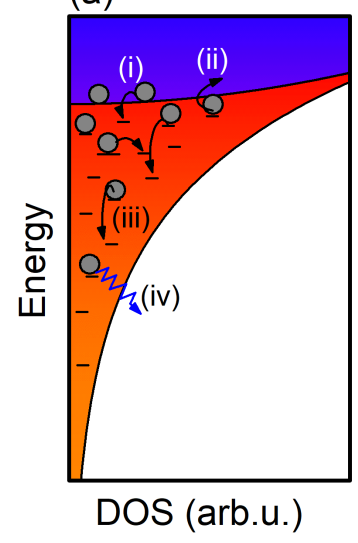

(b)

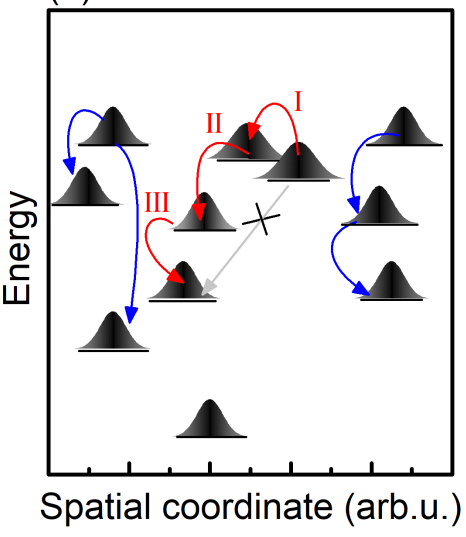

(c)

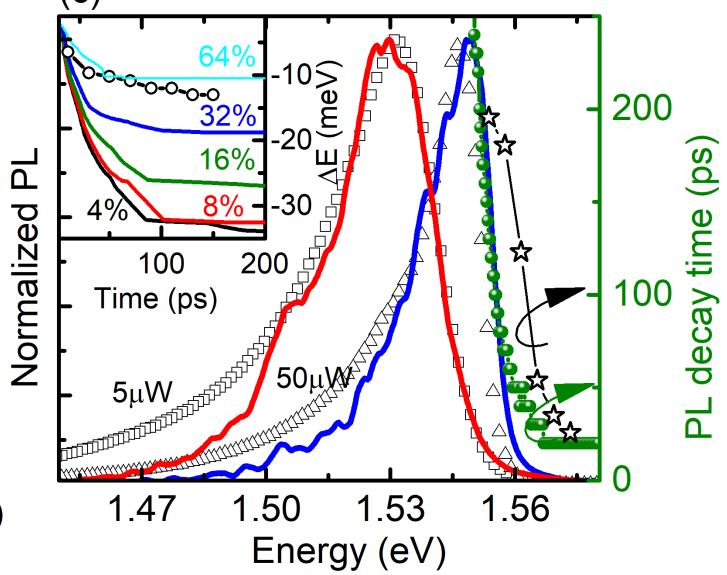

Figure 3: (a) Scheme of possible processes taken into account in the simulation of exciton kinetics: (i) exciton trapping, (ii) exciton activation to band states, (iii) exciton hopping, (iv) exciton recombination. The orange part represents the energy distribution of trapping states and the blue part is a continuum of extended states (free exciton density of states). (b) Blue arrows indicate allowed carrier paths at very low temperature, i.e., carriers can only relax to states nearby and only with a lower energy. Red arrows indicate possible paths at slightly higher temperature. In this case, the carriers can also hop to near states with slightly higher energy (I). Therefore they became more mobile and can reach more global minimum in the process indicated by (II) and (III). This can lead to the observation of an initial redshift with slightly increasing temperature (see Fig. 4(c) between 1.5-25 K). (c) Experimental PL spectra compared to predictions of the hopping model. The red and blue curves correspond to simulations performed with initial amount of injected carriers $8 \%$ and $32 \%$ of the number of trapping states to simulate different excitation powers. The squares and triangles are PL spectra measured at $5 \mu \mathrm{W}$ and $50 \mu \mathrm{W}$ excitation power. The green points correspond to simulated PL decay time dispersion. The stars are PL decay times determined experimentally. Inset: shift of the simulated PL peak position as a function of time for different trapping state saturation regimes together with experimental results (open points).

all reproduced with a good qualitative agreement and reasonable quantitative agreement.

Our model, combined with our experimental results, shows that at very low temperature the disorder and related shallow states present in organic-inorganic perovskites have a very similar impact on the PL as in case of classic inorganic semiconductors. The origin of potential fluctuation might be related to the point defects such as metal or halide vacancies, interstitials $^{69170}$ or alloying effect. The redshift of PL spectra related to light induced halide migration can be probably excluded since it is not efficient at low temperatures ${ }^{71}$ (see Supporting Information about stability of the investigated TC). The origin of the disorder can 

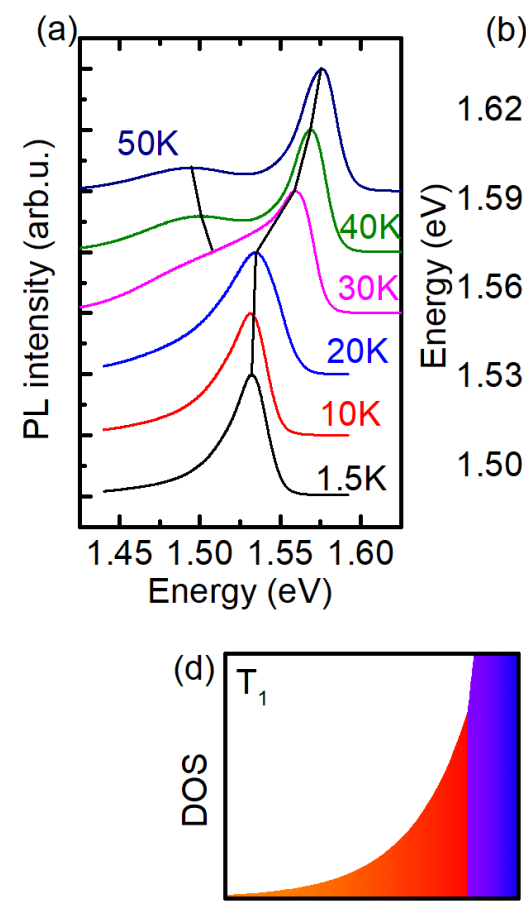
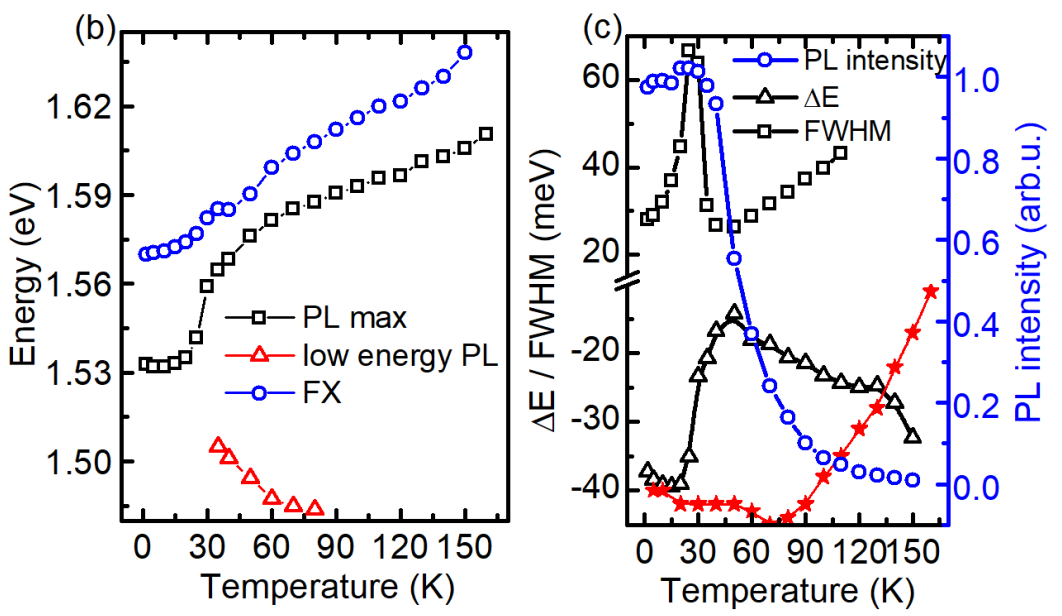

(f)

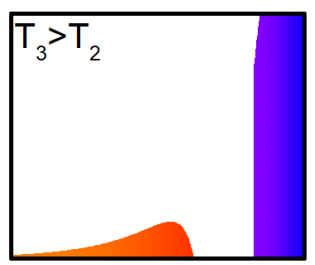

Energy

Figure 4: (a) Time integrated PL spectra measured at different temperatures. The black lines are a guide to the eye. (b) Temperature dependence of free exciton transition (blue circle), main PL peak (black squares), and low energy PL peak (red triangles), (c) Stokes shift $(\Delta \mathrm{E})$, PL broadening (FWHM), and PL intensity as a function of temperature. The red stars show results of Stokes shift simulation for an average energy of trapping states distribution of $14 \mathrm{meV}$. (d)-(f) Schematics of a possible evolution of the density of states with increasing temperature, which explains the splitting observed in the PL spectra (orange: localized states. Blue: extended states).

be also related to a different orientation of the frozen organic cations $\frac{12 \mid 1432}{34}$ and tetragonal phase inclusions, $\frac{35136}{36}$ which is supported by the temperature dependent PL measurements presented below.

Figure 4(a) shows the evolution of the PL spectra as a function of temperature. Initially, the main PL peak position exhibits a small (few meV) red shift with increasing temperature. Above $T=20 \mathrm{~K}$ a second feature appears at lower energies in the PL spectrum, while the main PL peak rapidly blue shifts (faster than the free exciton, determined via transmission measurements), which reduces the Stokes shift (see Fig. 4(b)). Initially, the additional feature appears as a shoulder increasing the FWHM before two clearly separated peaks can be 
observed above $30 \mathrm{~K}$ (see Fig. [4(a,c)). The Stokes shift is $\sim 38 \mathrm{meV}$ at $1.5 \mathrm{~K}$, and it decreases to around $15 \mathrm{meV}$ at $40-50 \mathrm{~K}$ (see Fig. 4(c)). In this so called "S-shape" behavior, 44172,75 the initial red shift of PL peak is usually understood as a redistribution (optimization) of the distribution of the excitons, which acquire enough thermal energy to leave their potential minima to find a lower energy state further afield. This behavior is correctly reproduced by the hopping model (see Supporting Information). With a further increase of temperature, according to the Miller-Abrahams formula, the motion of excitons between localized states becomes faster. This facilitates the establishment of a thermal distribution of the excitons, which in turn induces a blue shift. The temperature for the onset of the blue shift depends on the average energy $E_{0}$ of trapping states ${ }^{65}$ (see Supplementary Information) and it is given by $T \approx 0.55 E_{0} / k_{\mathrm{B}}$.

Based on the estimated value of $E_{0}=14 \mathrm{meV}$ from the low temperature $(1.5 \mathrm{~K})$ measurements, the onset of the blue shift (decrease of PL Stokes shift) should start at around $90 \mathrm{~K}$, which is a factor of three larger than the observed onset temperature of $\sim 30 \mathrm{~K}$. In fact, such a significant blue shift between $25 \mathrm{~K}$ and $50 \mathrm{~K}$ is difficult to explain only based on carrier redistribution or activation to free states. This suggests that an additional mechanism is at work, which we attribute to microstructural evolution of the perovskite crystal with a transition from static disorder to dynamic disorder. According to theoretical predictions, different orientations of the frozen organic cations $\sqrt{\frac{1214432}{34}}$ and tetragonal phase inclusions ${ }^{\frac{35136}{3}}$ can produce potential landscape fluctuations giving rise to the observed tail density of states emission at very low temperature. In the low temperature orthorombic phase of $\mathrm{MAPbI}_{3}$ or $\mathrm{FAPbI}_{3}$, one would expect that the organic cations are all aligned in the same direction, because of the constraints imposed by the $\mathrm{PbI}_{6}$ backbone. However, molecular dynamic calculations show that, upon cooling, the disordered configuration can also persist, which leads to a local bandgap modification and therefore to the formation of tail density of states.12 This disordered orthorhombic domains are metastable states, thus, an increase of temperature leads to the transition to more ordered alignment of organic cations, i.e., formation 
of ordered orthorhombic phase and vanishing tail density of states. Additionally, the activation of the motion of the organic cations with increasing temperature can also screen the potential of point defects,$\frac{69}{6 h i c h}$ effectively can be treated as a reduction of their density. The evolution of the density of trapping states is not only reflected by a rapid decrease of the Stokes shift, but also by the evolution of the PL line shape (Fig. 4(a)). The appearance of the second peak around $30 \mathrm{~K}$, which progressively red shifts with increasing temperature, points to the melting of frozen domains from shallow to deeper ones, as schematically illustrated in Fig. $4(\mathrm{~d}-\mathrm{f})$. The temperature evolution of the PL is qualitatively very similar to that of $\mathrm{MAPbI}_{3} \cdot{ }^{12}$ However, the temperature at which the melting of the frozen domain begin is lower in the case of the triple cation perovskite $(25 \mathrm{~K})$, as compared to $50 \mathrm{~K}$ for $\mathrm{MAPbI}_{3}$. Conversely, this temperature is much closer to that of pure $\mathrm{FAPbI}_{3}, \frac{47}{,}$ which is not surprising, considering the composition of the investigated TC perovskite. Also the low temperature FWHM of the PL spectrum of the TC perovskite film is similar to that reported for $\mathrm{FAPbI}_{3}{ }^{\frac{47766}{}}$ (around $30 \mathrm{meV}$ ), and significantly lower than in $\mathrm{MAPbI}_{3}, \stackrel{12176}{{ }^{12}}$ which can be attributed to the smaller dipole moment of formamidinium. 12

The Stokes shift does not vanish completely above $50 \mathrm{~K}$, which can indicate that the population of trapping states is not removed completely or that a new mechanism of excitons trapping is activated. Moreover, in contrast to III-V semiconductors, ${ }^{72}[4]$ the Stokes shift of the main PL peak begins to increase again at higher temperatures. The absolute value of the Stokes shift increases by a factor of two from $\simeq 15 \mathrm{meV}$ at $50 \mathrm{~K}$ to $\simeq 30 \mathrm{meV}$ at $150 \mathrm{~K}$. This non-vanishing and increasing Stokes shift at high temperatures may be the hallmark of the

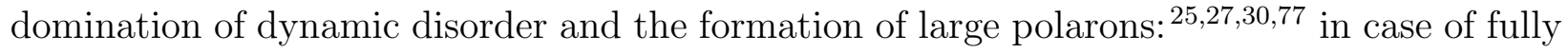
static disorder, we would expect a progressive decrease of the Stokes shift. The transmission measurement provides information about the energy of the nascent, bare exciton transition, while the emission originates from the electron and holes dressed by the polarization of organic cations or inorganic lattice deformation. The exact nature of the polaron formation in the orthorhombic phase of perovskites is not obvious. The reorientation of the whole 
organic dipole is not possible. However, it has been demonstrated that the motion of the organic cations is not completely frozen, even at very low temperatures,, 78 and possibly these remaining vibrations can play a role in the increasing Stokes shift observed above $50 \mathrm{~K}$. The temperature at which this Stokes shift increases corresponds very well to the temperature of the glassy transformation observed in $\mathrm{FAPbI}_{3}$ ( $\mathrm{FA}$ which dominates over other cations in our triple cations sample) $\stackrel{\sqrt[33]{3}}{\cdot}$ Moreover, recent work shows that the polaron formation can be also related to inorganic lattice vibration/deformation. ${ }^{29|79| 80}$ For example, the energy of the I-PbI bending mode is $21 \mathrm{~cm}^{-1}$, therefore we can expect that it is active even at low temperatures. However, precisely understanding what type of organic or inorganic sublattice motion can lead to the formation of polaron states at low temperatures requires additional extensive theoretical studies. The increasing value of the Stokes shift with increasing temperature (in the high temperature range) might be attributed to the activation of new vibrational modes, which can result in more efficient coupling of carriers with the lattice and therefore increasing value of Stokes shift.

The transition from a static disorder at very low temperature to a dynamic disorder at higher temperatures is accompanied by a significant drop of the PL emission intensity (see blue points in Fig. 4(c)). This can be attributed to the increased mobility of excitons or polarons (large polarons behave like free carriers with an increased effective mass) when the population of trapping states and fluctuations are reduced with the initial increase of temperature $(1.5-50 \mathrm{~K})$. It is well known that the PL efficiency and the concentration of nonradiative traps strongly varies from place to place in perovskite thin films. ${ }^{5}$ Therefore, we can expect that more mobile excitons/polarons have an enhanced probability to reach nonradiative recombination centers than excitons localized on traps induced by frozen disorder. A similar impact of the disorder on the PL efficiency has been reported in InGaN alloys. $\underline{81}$ 


\section{CONCLUSIONS}

In conclusion, we have systematically investigated the exciton dynamics in TC thin films using PL and transmission measurements. We show that at very low temperatures $(T<20 \mathrm{~K})$ the PL possesses the characteristic features of the emission observed in inorganic disordered semiconductors. The power dependence of the Stokes shift, the energy dependence of the decay time and the low energy tail of the PL spectrum all point to a disorder induced exponential tail in the density of states. A hopping model for excitons qualitatively explains all the characteristic features of the PL spectra from our organic-inorganic lead halide perovskite thin films at very low temperature. The estimated average energy of the trapping states is $14 \mathrm{meV}$. Unlike "classic" semiconductors, the temperature behavior of the PL indicates that the disorder and trapping state population evolve with increasing temperature. The character of disorder changes its nature from static to dynamic when lattice vibration couple with the carriers and probably lead to polaron formation. This is supported by a rapid decrease of Stokes shift and FWHM of PL, which cannot be explained by a simple carrier redistribution over the exponential tail in the density of states. The change of the disorder character is accompanied by a significant drop of the PL efficiency. We attribute this observation to the increased mobility of the excitons, which can reach more easily non-radiative recombination centers.

\section{ASSOCIATED CONTENT}

Details of hopping excitons model, power dependence of time resolved photoluminescence, XRD spectrum. 


\section{Acknowledgement}

This work was partially supported by the Région Midi-Pyrénées under contract MESR 13053031, BLAPHENE and STRABOT projects, which received funding from the IDEX Toulouse, Emergence program, "Programme des Investissements d'Avenir" under the program ANR-11-IDEX-0002-02, reference ANR-10-LABX-0037-NEXT. N. Z. holds a fellowship from the Chinese Scholarship Council (CSC). M.B. appreciates support from the Polish Ministry of Science and Higher Education within the Mobilnosc Plus program (grant no. 1648/MOB/V/2017/0). Z.A.-G. acknowledges funding from a Winton Studentship, and ICON Studentship from the Lloyds Register Foundation. S.D.S acknowledges the European Research Council (ERC) under the European Unions Horizon 2020 research and innovation

programme (HYPERION, grant agreement number 756962), and the Royal Society and Tata Group (UF150033). The work was supported by a Royal Society International Exchanges Cost Share award (IEC $\backslash \mathrm{R} 2 \backslash 170108)$.

\section{References}

(1) Saliba, M.; Matsui, T.; Domanski, K.; Seo, J.-Y.; Ummadisingu, A.; Zakeeruddin, S. M.; Correa-Baena, J.-P.; Tress, W. R.; Abate, A.; Hagfeldt, A. et al. Incorporation of Rubidium Cations into Perovskite Solar Cells Improves Photovoltaic Performance. Science 2016, 354, 206-209.

(2) Yang, W. S.; Park, B.-W.; Jung, E. H.; Jeon, N. J.; Kim, Y. C.; Lee, D. U.; Shin, S. S.; Seo, J.; Kim, E. K.; Noh, J. H. et al. Iodide Management in Formamidinium-LeadHalide-Based Perovskite Layers for Efficient Solar Cells. Science 2017, 356, 1376-1379.

(3) Chen, Y.; Zhang, L.; Zhang, Y.; Gao, H.; Yan, H. Large-Area Perovskite Solar Cells a Review of Recent Progress and Issues. RSC Adv. 2018, 8, 10489-10508.

(4) Stranks, S. D.; Eperon, G. E.; Grancini, G.; Menelaou, C.; Alcocer, M. J.; Leijtens, T.; 
Herz, L. M.; Petrozza, A.; Snaith, H. J. Electron-Hole Diffusion Lengths Exceeding 1 Micrometer in an Organometal Trihalide Perovskite Absorber. Science 2013, 342, $341-344$.

(5) de Quillettes, D. W.; Vorpahl, S. M.; Stranks, S. D.; Nagaoka, H.; Eperon, G. E.; Ziffer, M. E.; Snaith, H. J.; Ginger, D. S. Impact of Microstructure on Local Carrier Lifetime in Perovskite Solar Cells. Science 2015, 348, 683-686.

(6) Hutter, E. M.; Gélvez-Rueda, M. C.; Osherov, A.; Bulović, V.; Grozema, F. C.; Stranks, S. D.; Savenije, T. J. Direct-Indirect Character of the Bandgap in Methylammonium Lead Iodide Perovskite. Nature Materials 2017, 16, 115-120.

(7) Yang, S.; Fu, W.; Zhang, Z.; Chen, H.; Li, C.-Z. Recent Advances in Perovskite Solar Cells: Efficiency, Stability and Lead-Free Perovskite. Journal of Materials Chemistry A 2017, 5, 11462-11482.

(8) Stranks, S. D.; Snaith, H. J. Metal-Halide Perovskites For Photovoltaic and LightEmitting Devices. Nature Nanotechnology 2015, 10, 391-402.

(9) Brivio, F.; Butler, K. T.; Walsh, A.; Van Schilfgaarde, M. Relativistic Quasiparticle SelfConsistent Electronic Structure of Hybrid Halide Perovskite Photovoltaic Absorbers. Physical Review B 2014, 89, 155204.

(10) Even, J. Pedestrian Guide to Symmetry Properties of the Reference Cubic Structure of 3D All-Inorganic and Hybrid Perovskites. Journal of Physical Chemistry Letters 2015, 6, 2238-2242.

(11) Yu, Z. Effective-Mass Model and Magneto-Optical Properties in Hybrid Perovskites. Scientific Reports 2016, 6, 28576.

(12) Dar, M. I.; Jacopin, G.; Meloni, S.; Mattoni, A.; Arora, N.; Boziki, A.; Zakeeruddin, S. M.; Rothlisberger, U.; Grätzel, M. Origin of Unusual Bandgap Shift and Dual 
Emission in Organic-Inorganic Lead Halide Perovskites. Science Advances 2016, 2, e1601156.

(13) Chen, Y.; Yi, H.; Wu, X.; Haroldson, R.; Gartstein, Y.; Rodionov, Y.; Tikhonov, K.; Zakhidov, A.; Zhu, X.-Y.; Podzorov, V. Extended Carrier Lifetimes and Diffusion in Hybrid Perovskites Revealed by Hall Effect and Photoconductivity Measurements. Nature Communications 2016, \%, 12253.

(14) Chen, T.; Foley, B. J.; Ipek, B.; Tyagi, M.; Copley, J. R.; Brown, C. M.; Choi, J. J.; Lee, S.-H. Rotational Dynamics of Organic Cations in the $\mathrm{CH}_{3} \mathrm{NH}_{3} \mathrm{PbI}_{3}$ Perovskite. Physical Chemistry Chemical Physics 2015, 17, 31278-31286.

(15) Wang, T.; Daiber, B.; Frost, J. M.; Mann, S. A.; Garnett, E. C.; Walsh, A.; Ehrler, B. Indirect to Direct Bandgap Transition in Methylammonium Lead Halide Perovskite. Energy \& Environmental Science 2017, 10, 509-515.

(16) Etienne, T.; Mosconi, E.; De Angelis, F. Dynamical Origin of the Rashba Effect in Organohalide Lead Perovskites: A Key to Suppressed Carrier Recombination in Perovskite solar cells? The Journal of Physical Chemistry Letters 2016, 7, 1638-1645.

(17) Quarti, C.; Mosconi, E.; De Angelis, F. Structural and Electronic Properties of OrganoHalide Hybrid Perovskites From ab Initio Molecular Dynamics. Physical Chemistry Chemical Physics 2015, 17, 9394-9409.

(18) Panzer, F.; Li, C.; Meier, T.; Köhler, A.; Huettner, S. Impact of Structural Dynamics on the Optical Properties of Methylammonium Lead Iodide Perovskites. Advanced Energy Materials 2017, 7, 1700286.

(19) Ma, J.; Wang, L.-W. The Nature of Electron Mobility in Hybrid Perovskite $\mathrm{CH}_{3} \mathrm{NH}_{3} \mathrm{PbI}_{3}$. Nano Letters 2017, 17, 3646-3654. 
(20) Motta, C.; El-Mellouhi, F.; Kais, S.; Tabet, N.; Alharbi, F.; Sanvito, S. Revealing the Role of Organic Cations in Hybrid Halide Perovskite $\mathrm{CH}_{3} \mathrm{NH}_{3} \mathrm{PbI}_{3}$. Nature Communications 2015, 6, 7026 .

(21) Ma, J.; Wang, L.-W. Nanoscale Charge Localization Induced by Random Orientations of Organic Molecules in Hybrid Perovskite $\mathrm{CH}_{3} \mathrm{NH}_{3} \mathrm{PbI}_{3}$. Nano Letters 2014, 15, $248-$ 253.

(22) Kubicki, D. J.; Prochowicz, D.; Hofstetter, A.; Pechy, P.; Zakeeruddin, S. M.; Gratzel, M.; Emsley, L. Cation Dynamics in Mixed-Cation (MA) x (FA) 1-x PbI3 Hybrid Perovskites from Solid-State NMR. Journal of the American Chemical Society 2017, 139, 10055-10061.

(23) Azarhoosh, P.; McKechnie, S.; Frost, J. M.; Walsh, A.; Van Schilfgaarde, M. Research Update: Relativistic Origin of Slow Electron-Hole Recombination in Hybrid Halide Perovskite Solar Cells. APL Materials 2016, 4, 091501.

(24) Kepenekian, M.; Even, J. Rashba and Dresselhaus Couplings in Halide Perovskites: Accomplishments and Opportunities for Spintronics and Spin-Orbitronics. The Journal of Physical Chemistry Letters 2017, 8, 3362-3370.

(25) Zhu, X.-Y.; Podzorov, V. Charge carriers in Hybrid Organic-Inorganic Lead Halide Perovskites Might be Protected as Large Polarons. Journal of Physical Chemistry Letters 2015, 6, 4758-4761.

(26) Zhu, H.; Miyata, K.; Fu, Y.; Wang, J.; Joshi, P. P.; Niesner, D.; Williams, K. W.; Jin, S.; Zhu, X.-Y. Screening in Crystalline Liquids Protects Energetic Carriers in Hybrid Perovskites. Science 2016, 353, 1409-1413.

(27) Kang, B.; Biswas, K. Shallow Trapping vs. Deep Polarons in a Hybrid Lead Halide Perovskite, $\mathrm{CH}_{3} \mathrm{NH}_{3} \mathrm{PbI}_{3}$. Physical Chemistry Chemical Physics 2017, 19, 27184-27190. 
(28) Neukirch, A. J.; Nie, W.; Blancon, J.-C.; Appavoo, K.; Tsai, H.; Sfeir, M. Y.; Katan, C.; Pedesseau, L.; Even, J.; Crochet, J. J. et al. Polaron Stabilization by Cooperative Lattice Distortion and Cation Rotations in Hybrid Perovskite Materials. Nano Letters 2016, 16, 3809-3816.

(29) Miyata, K.; Meggiolaro, D.; Trinh, M. T.; Joshi, P. P.; Mosconi, E.; Jones, S. C.; De Angelis, F.; Zhu, X.-Y. Large Polarons in Lead Halide Perovskites. Science Advances 2017, 3, e1701217.

(30) Miyata, K.; Atallah, T. L.; Zhu, X.-Y. Lead Halide Perovskites: Crystal-Liquid Duality, Phonon Glass Electron Crystals, and Large Polaron Formation. Science Advances 2017, 3, e1701469.

(31) Poglitsch, A.; Weber, D. Dynamic Disorder in Methylammoniumtrihalogenoplumbates (II) Observed by Millimeter-Wave Spectroscopy. The Journal of Chemical Physics 1987, 87, 6373-6378.

(32) Leguy, A. M.; Frost, J. M.; McMahon, A. P.; Sakai, V. G.; Kockelmann, W.; Law, C.; Li, X.; Foglia, F.; Walsh, A.; ORegan, B. C. et al. The Dynamics of Methylammonium Ions in Hybrid Organic-Inorganic Perovskite Solar Cells. Nature Communications 2015, 6, 7124 .

(33) Fabini, D. H.; Hogan, T.; Evans, H. A.; Stoumpos, C. C.; Kanatzidis, M. G.; Seshadri, R. Dielectric and Thermodynamic Signatures of Low-Temperature Glassy Dynamics in the Hybrid Perovskites $\mathrm{CH}_{3} \mathrm{NH}_{3} \mathrm{PbI}_{3}$ and $\mathrm{HC}\left(\mathrm{NH}_{2}\right)_{2} \mathrm{PbI}_{3}$. Journal of Physical Chemistry Letters 2016, 7, 376-381.

(34) Mattoni, A.; Filippetti, A.; Saba, M.; Delugas, P. Methylammonium Rotational Dynamics in Lead Halide Perovskite by Classical Molecular Dynamics: the Role of Remperature. The Journal of Physical Chemistry C 2015, 119, 17421-17428. 
(35) Tahara, H.; Endo, M.; Wakamiya, A.; Kanemitsu, Y. Experimental Evidence of Localized Shallow States in Orthorhombic Phase of $\mathrm{CH}_{3} \mathrm{NH}_{3} \mathrm{PbI}_{3}$ Perovskite Thin Films Revealed by Photocurrent Beat Spectroscopy. The Journal of Physical Chemistry C 2016, 120, 5347-5352.

(36) Galkowski, K.; Mitioglu, A.; Surrente, A.; Yang, Z.; Maude, D.; Kossacki, P.; Eperon, G.; Wang, J.-W.; Snaith, H.; Plochocka, P. et al. Spatially Resolved Studies of the Phases and Morphology of Methylammonium and Formamidinium Lead Tri-Halide Perovskites. Nanoscale 2017, 9, 3222-3230.

(37) Soufiani, A. M.; Yang, Z.; Young, T.; Miyata, A.; Surrente, A.; Pascoe, A.; Galkowski, K.; Abdi-Jalebi, M.; Brenes, R.; Urban, J. et al. Impact of Microstructure on the Electron-Hole Interaction in Lead Halide Perovskites. Energy \& Environmental Science 2017, 10, 1358-1366.

(38) Saliba, M.; Matsui, T.; Seo, J.-Y.; Domanski, K.; Correa-Baena, J.-P.; Nazeeruddin, M. K.; Zakeeruddin, S. M.; Tress, W.; Abate, A.; Hagfeldt, A. et al. CesiumContaining Triple Cation Perovskite Solar Cells: Improved Stability, Reproducibility and High Efficiency. Energy \& Environmental Science 2016, 9, 1989-1997.

(39) Miyata, A.; Mitioglu, A.; Plochocka, P.; Portugall, O.; Wang, J. T.-W.; Stranks, S. D.; Snaith, H. J.; Nicholas, R. J. Direct Measurement of The Exciton Binding Energy and Effective Masses for Charge Carriers in Organic-Inorganic Tri-Halide Perovskites. Nature Physics 2015, 11, 582-587.

(40) Galkowski, K.; Mitioglu, A.; Miyata, A.; Plochocka, P.; Portugall, O.; Eperon, G. E.; Wang, J. T.-W.; Stergiopoulos, T.; Stranks, S. D.; Snaith, H. J. et al. Determination of the Exciton Binding Energy and Effective Masses for Methylammonium and Formamidinium Lead Tri-Halide Perovskite Semiconductors. Energy \& Environmental Science 2016, 9, 962-970. 
(41) Yang, Z.; Surrente, A.; Galkowski, K.; Bruyant, N.; Maude, D. K.; Haghighirad, A. A.; Snaith, H. J.; Plochocka, P.; Nicholas, R. J. Unraveling the Exciton Binding Energy and the Dielectric Constant in Single-Crystal Methylammonium Lead Triiodide Perovskite. The Journal of Physical Chemistry Letters 2017, 8, 1851-1855.

(42) Yang, Z.; Surrente, A.; Galkowski, K.; Miyata, A.; Portugall, O.; Sutton, R. J.; Haghighirad, A. A.; Snaith, H. J.; Maude, D. K.; Plochocka, P. et al. Impact of the Halide Cage on the Electronic Properties of Fully Inorganic Cesium Lead Halide Perovskites. ACS Energy Letters 2017, 2, 1621-1627.

(43) Henini, M. Dilute nitride semiconductors; Elsevier: Oxford U.K., 2004.

(44) Baranovskii, S.; Eichmann, R.; Thomas, P. Temperature-Dependent Exciton Luminescence in Quantum Wells by Computer Simulation. Physical Review B 1998, 58, 13081.

(45) Chichibu, S.; Sugiyama, M.; Onuma, T.; Kitamura, T.; Nakanishi, H.; Kuroda, T.; Tackeuchi, A.; Sota, T.; Ishida, Y.; Okumura, H. Localized Exciton Dynamics in Strained Cubic $\operatorname{In}_{0} .1 \mathrm{Ga}_{0} .9 \mathrm{~N} / \mathrm{GaN}$ Multiple Quantum Wells. Applied Physics Letters 2001, 79, 4319-4321.

(46) Skolnick, M.; Tapster, P.; Bass, S.; Pitt, A.; Apsley, N.; Aldred, S. Investigation of InGaAs-InP Quantum Wells by Optical Spectroscopy. Semiconductor Science and Technology 1986, 1, 29-40.

(47) Wright, A. D.; Milot, R. L.; Eperon, G. E.; Snaith, H. J.; Johnston, M. B.; Herz, L. M. Band-Tail Recombination in Hybrid Lead Iodide Perovskite. Advanced Functional Materials 2017, 27, 1700860.

(48) Buyanova, I.; Chen, W.; Pozina, G.; Bergman, J.; Monemar, B.; Xin, H.; Tu, C. Mechanism for Low-Temperature Photoluminescence in GaNAs/GaAs Structures Grown by Molecular-Beam Epitaxy. Applied Physics Letters 1999, 75, 501-503. 
(49) Mair, R.; Lin, J.; Jiang, H.; Jones, E.; Allerman, A.; Kurtz, S. Time-Resolved Photoluminescence Studies of $\operatorname{In}_{x} \mathrm{Ga}_{1-x} \mathrm{As}_{1-y} \mathrm{~N}_{y}$. Applied Physics Letters 2000, 76, 188-190.

(50) Kong, W.; Ye, Z.; Qi, Z.; Zhang, B.; Wang, M.; Rahimi-Iman, A.; Wu, H. Characterization of an Abnormal Photoluminescence Behavior Upon Crystal-Phase Transition of Perovskite $\mathrm{CH}_{3} \mathrm{NH}_{3} \mathrm{PbI}_{3}$. Physical Chemistry Chemical Physics 2015, 17, 16405-16411.

(51) Yang, J.; Wen, X.; Xia, H.; Sheng, R.; Ma, Q.; Kim, J.; Tapping, P.; Harada, T.; Kee, T. W.; Huang, F. et al. Acoustic-Optical Phonon Up-Conversion and Hot-Phonon Bottleneck in Lead-Halide Perovskites. Nature Communications 2017, 8, 14120.

(52) Bretschneider, S. A.; Laquai, F.; Bonn, M. Trap-Free Hot Carrier Relaxation in LeadHalide Perovskite Films. The Journal of Physical Chemistry C 2017, 121, 11201-11206.

(53) Fang, H.-H.; Adjokatse, S.; Shao, S.; Even, J.; Loi, M. A. Long-Lived Hot-Carrier Light Emission and Large Blue Shift in Formamidinium Tin Triiodide Perovskites. Nature Communications 2018, 9, 243.

(54) Shah, J. Ultrafast Spectroscopy of Semiconductors and Semiconductor Nanostructures; 2nd ed.; Springer: Berlin, 1999.

(55) Rubel, O.; Baranovskii, S.; Hantke, K.; Kunert, B.; Rühle, W.; Thomas, P.; Volz, K.; Stolz, W. Model of Temperature Quenching of Photoluminescence in Disordered Semiconductors and Comparison to Experiment. Physical Review B 2006, 73, 233201.

(56) Shakfa, M.; Wiemer, M.; Ludewig, P.; Jandieri, K.; Volz, K.; Stolz, W.; Baranovskii, S.; Koch, M. Thermal Quenching of Photoluminescence in Ga(AsBi). Journal of Applied Physics 2015, 117, 025709 .

(57) Kazlauskas, K.; Tamulaitis, G.; Pobedinskas, P.; Žukauskas, A.; Springis, M.; Huang, C.-F.; Cheng, Y.-C.; Yang, C. Exciton Hopping in $\operatorname{In}_{x} G_{a} a_{1-x} N$ Multiple Quantum Wells. Physical Review B 2005, 71, 085306. 
(58) Jandieri, K.; Kunert, B.; Liebich, S.; Zimprich, M.; Volz, K.; Stolz, W.; Gebhard, F.; Baranovskii, S.; Koukourakis, N.; Gerhardt, N. et al. Nonexponential Photoluminescence Transients in a Ga (NAsP) Tattice Matched to a (001) Silicon Substrate. Physical Review B 2013, 8\%, 035303.

(59) Baranowski, M.; Latkowska, M.; Kudrawiec, R.; Misiewicz, J. Model of Hopping Excitons in GaInNAs: Simulations of Sharp Lines in Micro-Photoluminescence Spectra and Their Dependence on the Excitation Power and Temperature. Journal of Physics: Condensed Matter 2011, 23, 205804.

(60) Baranowski, M.; Kudrawiec, R.; Latkowska, M.; Syperek, M.; Misiewicz, J.; Gupta, J. Dynamics of localized excitons in $\mathrm{Ga}_{0.69} \mathrm{In}_{0.31} \mathrm{~N}_{0.015} \mathrm{As}_{0.985} / \mathrm{GaAs}$ quantum well: Experimental Studies and Monte-Carlo Simulations. Applied Physics Letters 2012, 100, 202105.

(61) Baranowski, M.; Kudrawiec, R.; Luce, A.; Latkowska, M.; Yu, K.; Kuang, Y.; Misiewicz, J.; Tu, C.; Walukiewicz, W. Temperature Evolution of Carrier Dynamics in $\mathrm{GaN}_{x} \mathrm{P}_{y} \mathrm{As}_{1-y-x}$ Alloys. Journal of Applied Physics 2015, 117, 175702.

(62) Miller, A.; Abrahams, E. Impurity Conduction at Low Concentrations. Physical Review 1960, 120, 745.

(63) Baranowski, M.; Kudrawiec, R.; Misiewicz, J. Theoretical Studies of the Influence of Temperature on Photoluminescence Dynamics in GaInNAs/GaAs Quantum Wells. Japanese Journal of Applied Physics 2013, 52, 08JL04.

(64) Baranowski, M.; Kudrawiec, R.; Misiewicz, J.; Hammar, M. Nitrogen-Related Changes in Exciton Localization and Dynamics in GaInNAs/GaAs Quantum Wells Grown by Metalorganic Vapor Phase Epitaxy. Applied Physics A 2015, 118, 479-486.

(65) Rubel, O.; Galluppi, M.; Baranovskii, S.; Volz, K.; Geelhaar, L.; Riechert, H.; Thomas, P.; Stolz, W. Quantitative Description of Disorder Parameters in (GaIn)(NAs) 
Quantum Wells from the Temperature-Dependent Photoluminescence Spectroscopy. Journal of Applied Physics 2005, 98, 063518.

(66) Baranowski, M.; Latkowska, M.; Kudrawiec, R.; Misiewicz, J. Hopping Excitons in GaInNAsSimulation of Micro-and Macro-photoluminescence Spectra. Acta Physica Polonica A 2011, 120, 899-901.

(67) Zhang, W.; Pathak, S.; Sakai, N.; Stergiopoulos, T.; Nayak, P. K.; Noel, N. K.; Haghighirad, A. A.; Burlakov, V. M.; Sadhanala, A.; Li, W. et al. Enhanced Optoelectronic Quality of Perovskite Thin Films with Hypophosphorous Acid for Planar Heterojunction Solar Cells. Nature Communications 2015, 6, 10030.

(68) De Wolf, S.; Holovsky, J.; Moon, S.-J.; Loper, P.; Niesen, B.; Ledinsky, M.; Haug, F.J.; Yum, J.-H.; Ballif, C. Organometallic Halide Perovskites: Sharp Optical Absorption Edge and Its Relation to Photovoltaic Performance. The Journal of Physical Chemistry Letters 2014, 5, 1035-1039.

(69) Nan, G.; Zhang, X.; Abdi-Jalebi, M.; Andaji-Garmaroudi, Z.; Stranks, S. D.; Lu, G.; Beljonne, D. How Methylammonium Cations and Chlorine Dopants Heal Defects in Lead Iodide Perovskites. Advanced Energy Materials 2018, 1702754.

(70) Chen, C.; Hu, X.; Lu, W.; Chang, S.; Shi, L.; Li, L.; Zhong, H.; Han, J.-B. Elucidating the Phase Transitions and Temperature-Dependent Photoluminescence of $\mathrm{MAPbBr}_{3}$ Single Crystal. Journal of Physics D: Applied Physics 2018, 51, 045105.

(71) Hoke, E. T.; Slotcavage, D. J.; Dohner, E. R.; Bowring, A. R.; Karunadasa, H. I.; McGehee, M. D. Reversible Photo-Induced Trap Formation in Mixed-Halide Hybrid Perovskites for Photovoltaics. Chemical Science 2015, 6, 613-617.

(72) Kaschner, A.; Lüttgert, T.; Born, H.; Hoffmann, A.; Egorov, A. Y.; Riechert, H. Recombination Mechanisms in GaInNAs/GaAs Multiple Quantum Wells. Applied Physics Letters 2001, 78, 1391-1393. 
(73) Cho, Y.-H.; Gainer, G.; Fischer, A.; Song, J.; Keller, S.; Mishra, U.; DenBaars, S. Sshaped Temperature-Dependent Emission Shift and Carrier Dynamics in InGaN/GaN Multiple Quantum Wells. Applied Physics Letters 1998, 73, 1370-1372.

(74) Bergman, L.; Dutta, M.; Stroscio, M.; Komirenko, S.; Nemanich, R. J.; Eiting, C.; Lambert, D.; Kwon, H.; Dupuis, R. Photoluminescence and Recombination Mechanisms in GaN/ $\mathrm{Al}_{0.2} \mathrm{Ga}_{0.8} \mathrm{~N}$ Superlattice. Applied Physics Letters 2000, 76, 1969-1971.

(75) Kopaczek, J.; Linhart, W.; Baranowski, M.; Richards, R.; Bastiman, F.; David, J.; Kudrawiec, R. Optical properties of GaAsBi/GaAs quantum wells: Photoreflectance, photoluminescence and time-resolved photoluminescence study. Semiconductor Science and Technology 2015, 30, 094005.

(76) Wright, A. D.; Verdi, C.; Milot, R. L.; Eperon, G. E.; Pérez-Osorio, M. A.; Snaith, H. J.; Giustino, F.; Johnston, M. B.; Herz, L. M. Electron-Phonon Coupling in Hybrid Lead Halide Perovskites. Nature Communications 2016, 7, 11755.

(77) Gong, J.; Yang, M.; Ma, X.; Schaller, R. D.; Liu, G.; Kong, L.; Yang, Y.; Beard, M. C.; Lesslie, M.; Dai, Y. et al. Electron-Rotor Interaction in Organic-Inorganic Lead Iodide Perovskites Discovered by Isotope Effects. The Journal of Physical Chemistry Letters 2016, 7, 2879-2887.

(78) Drużbicki, K.; Pinna, R. S.; Rudić, S.; Jura, M.; Gorini, G.; Fernandez-Alonso, F. Unexpected Cation Dynamics in the Low-Temperature Phase of Methylammonium Lead Iodide: the Need for Improved Models. The Journal of Physical Chemistry Letters 2016, 7, 4701-4709.

(79) Park, M.; Neukirch, A. J.; Reyes-Lillo, S. E.; Lai, M.; Ellis, S. R.; Dietze, D.; Neaton, J. B.; Yang, P.; Tretiak, S.; Mathies, R. A. Excited-state Vibrational Dynamics Toward the Polaron in Methylammonium Lead Iodide Perovskite. Nature Communications 2018, 9, 2525. 
(80) Ambrosio, F.; Wiktor, J.; De Angelis, F.; Pasquarello, A. Origin of Low Electron-Hole Recombination Rate in Metal Halide Perovskites. Energy 83 Environmental Science 2018, 11, 101-105.

(81) Nakamura, S. The Roles of Structural Imperfections in InGaN-Based Blue LightEmitting Diodes and Laser Diodes. Science 1998, 281, 956-961. 


\section{Graphical TOC Entry}

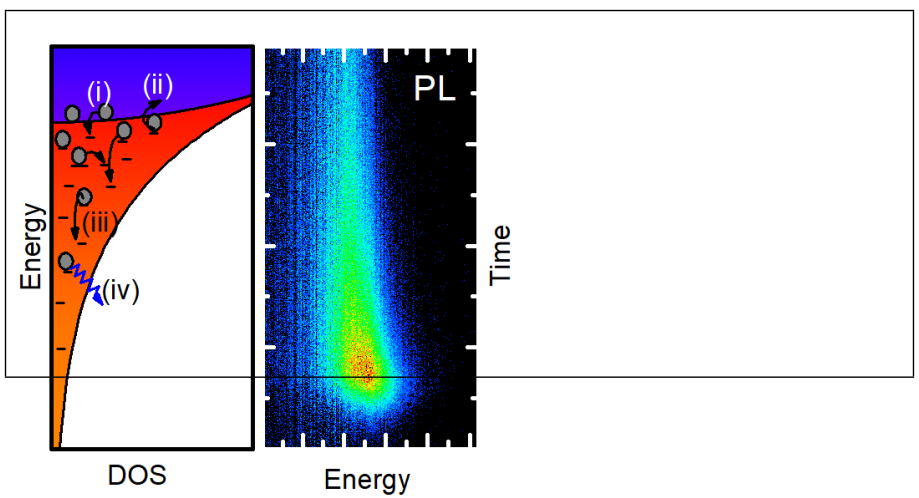

